Chris Freiling, Department of Mathematics, California State University, San

Bernardino, CA 92407. e-mail: cfreiling@csusb.edu

\title{
THE EQUIVALENCE OF UNIVERSAL AND ORDINARY FIRST-RETURN DIFFERENTIATION
}

\begin{abstract}
If a function $F(x)$ is first-return differentiable to $f(x)$ then it is also universally first-return differentiable to $f(x)$.
\end{abstract}

We show that if a function $F: \mathbb{R} \rightarrow \mathbb{R}$ has a first-return derivative $f(x)$ then in fact it is universally first return differentiable to the same function $f(x)$. This answers the second of two questions raised by M.J. Evans at the 1994 Łódź conference workshop. We note in Evans' original question, $F$ : $[0,1] \rightarrow \mathbb{R}$. (One might also consider $F:(0,1) \rightarrow \mathbb{R}$.) For convenience, we assume $F: \mathbb{R} \rightarrow \mathbb{R}$, but all three versions of the theorem are easily seen to be equivalent (see [4]). In contrast, Darji, Evans, and O'Malley have characterized the first-return continuous functions as those which are Darboux and Baire 1 (see [2]) while their characterization of the universally first-return continuous functions turns out to be a proper subclass of this (see [1], [3]).

We first recall some terminology and introduce some notation. Let $S$ be a countable dense set of reals, which we call the "support set". Let $\sigma: S \rightarrow \mathbb{Z}^{+}$ be an injection, or an ordering on $S$, which is referred to as a "trajectory". For each $s \in S$, we call $\sigma(s)$ the $\operatorname{rank}$ of $s($ or $\operatorname{rank}(s))$. The "path system" $P$ denotes the relation on $S \times \mathbb{R}$ defined by $(s, x) \in P$ iff $s \neq x$ and no element $r \in S$ between $s$ and $x$ has $\operatorname{rank}(r)<\operatorname{rank}(s)$. For each real number $x$, let $\operatorname{path}(x)$ denote the set $\{s \in S \mid(s, x) \in P\}$ and conversely, for each $s \in S$ let range $(s)$ denote the set $\{x \mid(s, x) \in P\}$. Note that range $(s)$ is always a closed neighborhood of $s$ with one point, $s$, removed. Most of the time we will want to talk about this range with the point $s$ included. In that case we will call it $\operatorname{Range}(s)=\operatorname{range}(s) \cup\{s\}$.

\footnotetext{
Key Words: differentiation, first-return

Mathematical Reviews subject classification: 26A24

Received by the editors September 12, 1994 and in revised form July 2, 2000

* The author would like to especially thank the editor, Mike Evans, and the referees for their heroic patience and understanding, for their generous help on this difficult article, and for catching many errors and oversights in the original draft.
} 
The limiting process as $y \rightarrow x, y \in \operatorname{path}(x)$ is called the " $\sigma$-first-return limit". Fix a real function $F$ and denote $(F(y)-F(x)) /(y-x)$ by $D(y, x)$. Then the " $\sigma$-first-return derivative" of $F$ at $x$ simply means the $\sigma$-first-return limit of $D(y, x)$. Note that the existence and the value of this derivative depends on the trajectory $\sigma$. We say $F$ is "first-return differentiable" to a finite function $f(x)$ if there exists some support set $S$ and some trajectory $\sigma: S \rightarrow \mathbb{Z}^{+}$such that for each $x$, the $\sigma$-first-return derivative of $F$ at $x$ is $f(x)$. We say that $F$ is "universally first-return differentiable" to $f(x)$ if given any countable dense set $T$ (called the "target set") there exists some trajectory $\tau: T \rightarrow \mathbb{Z}^{+}$such that at each $x$, the $\tau$-first-return derivative of $F$ is $f(x)$.

We will prove the following theorem

Theorem 1. If $F(x): \mathbb{R} \rightarrow \mathbb{R}$ is first-return differentiable to a finite function $f(x)$, then $F(x)$ is also universally first-return differentiable to $f(x)$.

We will prove this theorem in a sequence of definitions and lemmas. Let $F$ be first-return differentiable to $f$. Let $S, \sigma, T$ be as above. Our goal is to find an appropriate trajectory $\tau$.

To avoid confusion, we will say "path" and "range" when we are referring to the trajectory $\sigma$. Later, when we need to refer to the trajectory $\tau$ we will use the terms "newpath" and "newrange". For $A \subseteq \mathbb{R}$ we let $\operatorname{cl}(A)$ denote the closure of $A, \operatorname{int}(A)$ denote the interior of $A$, and $\mathrm{c} A$ denote the complement of $A$.

Definition 2. For each pair of positive integers $m, n$ we let

$$
X_{m, n}=\{x|(\operatorname{rank}(s) \geq m, s \in \operatorname{path}(x)) \rightarrow| D(s, x)-f(x) \mid<1 / n\} .
$$

The following proposition follows immediately.

Proposition 3. If $m^{\prime} \geq m$ and $n^{\prime} \leq n$, then $X_{m, n} \subset X_{m^{\prime}, n^{\prime}}$. Also, $F$ is first-return differentiable to $f(x)$ means precisely that for each $n \in \mathbb{Z}^{+}$, $\cup_{m} X_{m, n}=\mathbb{R}$.

The following simple fact is used often enough that we list it as a lemma. When we need this fact, we will simply refer to it as "Convexity".

Lemma 4. (Convexity) Suppose that $u<v<w$. Then $D(u, w)$ is between $D(u, v)$ and $D(v, w)$ (inclusive).

ProOF.

$$
D(u, w)=(D(u, v)(v-u)+D(v, w)(w-v)) /(w-u)
$$


is a convex combination of $D(u, v)$ and $D(v, w)$.

Then next lemma is very similar and serves as a partial converse to the previous lemma.

Lemma 5. Let $v$ be any number between $u$ and $w$, which is closer to $u$ than it is to $w$. Suppose that both $D(u, w)$ and $D(u, v)$ are both within $\epsilon$ of some number $y$. Then $D(v, w)$ is within $3 \epsilon$ of $y$.

Proof.

$$
\begin{aligned}
D(v, w) & =(D(u, w) \cdot(w-u)-D(u, v) \cdot(v-u)) /(w-v) \\
D(v, w)- & =((D(u, w)-y) \cdot(w-u)-(D(u, v)-y) \cdot(v-u)) /(w-v) \\
|D(u, w)-y| & <(\epsilon \cdot(w-u)+\epsilon \cdot(v-u)) /(w-v) \\
& =\epsilon \cdot((w-u)+(v-u)) /((w-u)-(v-u)) .
\end{aligned}
$$

Then, since $|v-u|<(1 / 2)|w-u|$, we have that $|D(u, w)-y|<3 \epsilon$.

The next lemma is the main principle which will lay the foundation of our construction of $\tau$.

Lemma 6. Given any $m \in \mathbb{Z}^{+}$and $r \in \mathbb{R}$ there is a neighborhood I of $r$ such that for each $n \leq m$ and each $x$ in $X_{m, n} \cap I$,

(i) $f(x)$ and $f(r)$ differ by less than $4 / n$;

(ii) if $x \neq r$, then $D(x, r), f(x)$ differ by less than $5 / n$;

(iii) if $x, y$ are both in $X_{m, n} \cap I$, then $f(x), f(y)$ differ by less than $8 / n$; and if, in addition, $x \neq y$, then $D(x, y), f(x)$ differ by less than $13 / n$.

Proof. Let $m^{\prime}$ be large enough that $m^{\prime}>m$ and $r \in X_{m^{\prime}, m}$. Let $u<r$ be such that $u \in \operatorname{path}(r)$ with $\operatorname{rank}(u)>m^{\prime}$. Let $n \leq m$ and suppose $x \in X_{m, n} \cap((u+r) / 2, r)$. Let $p$ be the element of $S$ with smallest rank between $x$ and $r$. Then $u<x<p<r$, and since $u \in \operatorname{path}(r), \operatorname{rank}(p)>\operatorname{rank}(u)>$ $m^{\prime}>m$. Also, since $u \in \operatorname{path}(r)$ we have $u \in \operatorname{path}(x)$. It follows from the definition of $X_{m, n}$ that $D(u, x), f(x)$ differ by less than $1 / n$. Furthermore, $p \in \operatorname{path}(x) \cap \operatorname{path}(r)$. Since $x \in X_{m, n}$, it follows that $D(x, p), f(x)$ differ by less than $1 / n$, as do $D(p, r), f(r)$. By convexity, $D(u, p)$ also differs from $f(x)$ by less than $1 / n$. Since $r \in X_{m^{\prime}, m} \subseteq X_{m^{\prime}, n}$ and $\operatorname{rank}(p) \geq m^{\prime}$, we also have that $D(p, r), f(r)$ differ by less than $1 / n$. Similarly, $D(u, r), f(r)$ differ by less than $1 / n$. Then since $(r-p)<(1 / 2)(r-u)$ we have from Lemma 5 , 
that $|D(u, p)-f(r)|<3 / n$. Since $D(u, p), f(x)$ differ by less than $1 / n$, we also get that $f(r), f(x)$ differ by less than $4 / n$.

Next, since $D(p, r)$ differs from $f(r)$ by less than $1 / n$, it differs from $f(x)$ by less than $5 / n$. Since we have already established that $D(x, p)$ differs from $f(x)$ by less than $1 / n$, it follows by convexity that $D(x, r), f(x)$ differ by less than $5 / n$. By a similar argument, there is a $v>r$ such that if $x \in$ $X_{m, n} \cap(r,(v+r) / 2)$, then $f(r), f(x)$ differ by less than $4 / n$ and $D(x, r), f(x)$ differ by less than $5 / n$. Therefore, letting $I=((u+r) / 2,(v+r) / 2)$, properties (i) and (ii) are established.

The first part of (iii) follows directly from (i). To see the second part, choose $p \in S$ of smallest rank between $x$ and $y$ so that $p \in \operatorname{path}(\mathrm{x}) \cap \operatorname{path}(y)$. Now $I$ was chosen small enough that, except for possibly $r$, all elements in $S \cap I$ have rank greater than $m$. Therefore, if $p \neq r$, then $\operatorname{rank}(p)>m$ so $D(x, p), f(x)$ differ by less than $1 / n$ as do $D(p, y), f(y)$. Since $f(x), f(y)$ differ by less than $8 / n$, we get $D(p, y), f(x)$ differ by less than $9 / n$. Hence, by convexity, $D(x, y), f(x)$ differ by less than $9 / n$. On the other hand, if $p=r$, then if $r$ is between $x$ and $y$. By (ii), $D(x, r), f(x)$ differ by less than $5 / n$, as do $D(r, y), f(y)$. Then $D(r, y), f(x)$ differ (using the first part of (iii)) by less than $13 / n$. Then, by convexity, $D(x, y), f(x)$ differ by less than $13 / n$.

Corollary 7. $F$ is continuous on the closure of each $X_{m, n}$.

Proof. We may assume without loss of generality that $n=1$, since $X_{m, n} \subseteq$ $X_{m, 1}$, and so $n \leq m$. Let $r \in \operatorname{cl}\left(X_{m, n}\right)$, and $I$ be as in Lemma 6. If $x \in X_{m, n} \cap I$ and $x \neq r$, then by Lemma 6 , (i) and (ii), $D(x, r)$ and $f(r)$ differ by less than $9 / n ;$ so $|D(x, r)|<|f(r)|+9 / n$. Then $|F(x)-F(r)|<|x-r|(|f(r)|+9 / n)$ and hence

$$
\lim _{x \rightarrow r, x \in X_{m, n}} F(x)=F(r) .
$$

Now let $\epsilon>0$, let $x \in \operatorname{cl}\left(X_{m, n}\right), x \neq r$, and $x$ close enough to $r$ so that any $x^{\prime} \in X_{m, n}$ within $2|x-r|$ of $r$ has $\left|F\left(x^{\prime}\right)-F(r)\right|<\epsilon / 2$. Using (1) again, we can choose an $x^{\prime} \in X_{m, n}$ arbitrarily close to $x$, with $\left|F\left(x^{\prime}\right)-F(x)\right|<\epsilon / 2$. It follows that $|F(x)-F(r)|<\epsilon$.

Corollary 8. If I is compact, then $F$ is bounded on $\operatorname{cl}\left(X_{m, n}\right) \cap I$.

The next corollary follows immediately from the fact, proved in [2], that a first-return differentiable function is universally first-return continuous. For convenience, we provide an alternate proof. 
Corollary 9. Let $I$ be any open neighborhood of $x$ and $J$ be any open neighborhood of $F(x)$. Then for some $t \in T \cap I$ we have $F(t) \in J$.

Proof. Let $A=\{x \in I \mid F(x) \in J\}$. We must show that $A \cap T \neq \phi$. Since $T$ is dense, it is enough to show that $A$ contains a nonempty open interval. By Proposition $3 \operatorname{cl}(A) \subseteq \cup_{m \in \mathbb{Z}^{+}} X_{m, 1}$; so by the Baire Category Theorem there is an open subinterval $K \subset I$ and a positive integer $m$ such that $K \cap A \neq \phi$ and $X_{m, 1}$ is dense in $\operatorname{cl}(A) \cap K$. Then, by Corollary $7, F$ is continuous on $\operatorname{cl}(A) \cap K$. Let $r \in A \cap K$. Then $F(r) \in J$. Then there must be some neighborhood $L \subset K$ of $r$ where each $p \in \operatorname{cl}(A) \cap L$ also has $F(p) \in J$. But then $\operatorname{cl}(A) \cap L \subset A \cap L$ so that $\mathrm{A}$ is closed in $L$.

Since $F$ is first-return differentiable, given any $x$ we can find points $s \in S$ which are arbitrarily close to $x$ on either side, such that $F(s)$ is arbitrarily close to $F(x)$. It follows that A has no points isolated on either side. Therefore, $L \subseteq A$.

Definition 10. Let $s \in S$ with bounded range, with rank $\geq m$. Let $r \in$ Range $(s)$. We say that $r$ is an $(m, n)$-good replacement for $s$ if and only if for some $\eta<16 / n$ if $x \in X_{m, n} \cap \operatorname{Range}(s)$ with $x \neq r$, then $|D(r, x)-f(x)|<\eta$.

Intuitively, $r$ performs almost as well as $s$ as an element of the support set. Note that if Range $(s)$ is bounded and $\operatorname{rank}(s) \geq m$, then by definition of $X_{m, n}, s$ is $(m, n)$-good for itself.

Definition 11. We say that $r$ is an m-good replacement for $s$ iff $r$ is an $(m, n)$-good replacement for $s$ for each $n \leq m$.

Corollary 12. For each $r \notin S, m \in \mathbb{Z}^{+}$, there are elements $s \in S$ arbitrarily close to $r$ such that $r$ is an $m$-good replacement for $s$.

Proof. Let I be as in Lemma 6 . Then by Lemma 6 (ii), any $s \in$ path $(r) \cap I$ with bounded range, $\operatorname{Range}(s) \subseteq I$, and $\operatorname{rank}(s) \geq m$ will suffice.

Lemma 13. The Theorem holds when $S \subset T$.

Proof. Let $\left\{t_{1}, t_{2}, \ldots\right\}$ be the elements of $T \backslash S$. Using Corollary 12 , let $\pi\left(t_{i}\right)$ denote the $s \in S$ of least rank such that $t_{i}$ is an $i$-good replacement for $s$ and $s \neq \pi\left(t_{j}\right)$ for $j<i$. If $t \in S$ let $\pi(t)=t$. Let $\tau: T \rightarrow \mathbb{Z}^{+}$by $\tau(t)=2 \sigma(\pi(t))+1$ if $t \notin S$ and $2 \sigma(\pi(t))$ if $t \in S$. Note that $\tau$ is one-to-one, preserves the order on $S$ induced by the trajectory $\sigma$, and that $\tau(\pi(t)) \leq \tau(t)$. The trajectory $\tau$ defines a new path system.

Claim: If $t \in \operatorname{newpath}(x)$ and $\pi(t) \neq x$, then $\pi(t) \in \operatorname{path}(x)$. 
Proof of Claim. Let $s \in S$ be between $\pi(t)$ and $x$. We must show $\sigma(s)>$ $\sigma(\pi(t))$. If $s=t$, then $t \in S$; so $\pi(t)=t$; so $s=\pi(t)$ which contradicts that $s$ is between $\pi(t)$ and $x$. Therefore, $s \neq t$ and so either $s$ is between $\pi(t), t$ or between $t, x$. If $s$ is between $\pi(t), t$, then by definition of $\pi, t$ is an $i$-good replacement for $\pi(t)$. In particular, $t \in \operatorname{Range}(\pi(t))$ and therefore, $\sigma(s)>\sigma(\pi(t))$. If $s$ is between $t, x$, then since $t \in \operatorname{newpath}(x)$ we have $\tau(s)>\tau(t) \geq \tau(\pi(t))$. But then $\sigma(s)>\sigma(\pi(t))$ which finishes the proof of the claim.

Fix $n, x$. We must show that if $t \in \operatorname{newpath}(x)$ with $\tau(t)$ large enough, then $D(t, x), f(x)$ differ by less than $1 / n$. Choose $m>16 n$ so large that $x \in X_{m, 16 n}$. First, consider $t_{i} \in T \backslash S$ with $t_{i} \in \operatorname{newpath}(x)$. Since $\pi$ is one-to-one on $T \backslash S$, $\pi\left(t_{i}\right)=x$ for at most one value of $i$. Therefore, choose $t_{i} \in$ newpath $(x)$ with $\tau\left(t_{i}\right)$ large enough to force $\pi\left(t_{i}\right) \neq x$, and also large enough that $i>m$, and $\tau\left(t_{i}\right)>2 m+1$. Then $\pi\left(t_{i}\right) \in \operatorname{path}(x)$ with $\sigma\left(\pi\left(t_{i}\right)\right)>m$. Since $t_{i}$ is an $i$-good replacement for $\pi\left(t_{i}\right)$ and $x \in X_{m, 16 n} \subseteq X_{i, 16 n}$, we have that $D\left(t_{i}, x\right), f(x)$ differ by less than $16 / 16 n=1 / n$. Next, consider $t \in S$ with $t \in \operatorname{newpath}(x)$ and with $\tau(t)>2 m$. Then $\pi(t)=t \in \operatorname{path}(x)$ and $\sigma(t)>m$. Since $x \in X_{m, 16 n}$ it follows that $D(t, x), f(x)$ differ by less than $1 / 16 n<1 / n$.

Definition 14. We say that $r$ is $(m, n)$-very good replacement for $s$ iff whenever $t$ is sufficiently close to $r$ and $F(t)$ is sufficiently close to $F(r)$, we have that $t$ is an $(m, n)$-good replacement for $s$.

Lemma 15. If $r \in \operatorname{int}(\operatorname{Range}(s)) \backslash c l\left(X_{m, n}\right)$ and $r$ is an $(m, n)$-good replacement for $s$, then $r$ is an $(m, n)$-very good replacement for $s$.

Proof. We must show that for any $t$ close enough to $r$, with $F(t)$ close enough to $F(r), t$ will be an $(m, n)$-good replacement for $s$. The first requirement on $t$ is to choose it close enough to $r$ so that $t \in \operatorname{Range}(s) \backslash \operatorname{cl}\left(X_{m, n}\right)$.

Our goal is to make sure that for any $x \in X_{m, n} \cap \operatorname{Range}(s), D(t, x)$ can be made arbitrarily close to $D(r, x)$ by simply choosing $t$ close enough to $r$ and $F(t)$ close enough to $F(r)$, and we want these closeness criteria to be independent of the particular choice of $x$. To see that this can be accomplished, let $d$ denote the distance from $r$ to $X_{m, n}$ and first choose $t$ so close to $r$ that the distance from $t$ to $X_{m, n}$ is greater than $d / 2$. Using Corollary 8, and the fact that $s$ has bounded range, let $B$ be any fixed number larger than $|F(r)|$ and $|F(x)|$ for all $x \in X_{m, n} \cap$ Range $(s)$. Note that

$$
\begin{aligned}
|D(t, x)-D(r, x)| & =\left|\frac{F(t)-F(x)}{t-x}-\frac{F(r)-F(x)}{r-x}\right| \\
& =\left|\frac{F(t)}{t-x}-\frac{F(r)}{t-x}+\frac{F(r)}{t-x}-\frac{F(r)}{r-x}+\frac{F(x)}{r-x}-\frac{F(x)}{t-x}\right|
\end{aligned}
$$




$$
\begin{aligned}
& \leq\left|\frac{F(t)-F(r)}{t-x}\right|+|F(r)-F(x)|\left|\frac{1}{t-x}-\frac{1}{r-x}\right| \\
& <\frac{|F(t)-F(r)|}{d / 2}+2 B\left|\frac{r-t}{d^{2} / 2}\right|
\end{aligned}
$$

which can be made arbitrarily small, just by choosing $|F(t)-F(r)|$ and $|r-t|$ small.

Lemma 16. Let $s$ be an element of $S$ with $\operatorname{rank}(s) \geq m$ and with bounded range. Let $I$ be a neighborhood of $s$ found by applying Lemma 6 to $s, m$. Then there is a neighborhood $J \subseteq I$ of $s$, with $\operatorname{cl}(J) \subset \operatorname{int}(\operatorname{Range}(s))$, such that for any $n \leq m$, if:

(i) $r \in J \cap \operatorname{cl}\left(X_{m, n}\right)$, or

(ii) $r \in J \cap S$ and $D(r, s), f(s)$ differ by at most 9, and for every $x \notin$ Range $(r)$ there is an $x^{\prime} \in X_{m, n} \cap \operatorname{Range}(r)$ between $r$ and $x$,

then $r$ is an $(m, n)$-good replacement for $s$.

Proof. Assume without loss of generality that $r \neq s$. Decrease I, if necessary, so that $\operatorname{cl}(I) \subset \operatorname{int}(\operatorname{Range}(s))$ and also so that every such $r \in S \cap I$ has $\operatorname{rank}(r) \geq m$. The condition that $D(r, s), f(s)$ differ by at most 9 , implies that $F(r)$ is close to $F(s)$, how close depends on the size of $J$. Choose $J$ so small that whenever $r \in J$, it is so close to $s$ with $F(r)$ so close to $F(s)$ that whenever $x \in \operatorname{cl}\left(X_{m, 1}\right) \cap$ Range $(s) \backslash I$, then $D(r, x), D(s, x)$ differ by less than $1 / \mathrm{m}$. This is made possible by Corollary 8 . This completes the choice of the interval $J$.

Let $n \leq m$ and let $r$ satisfy either (i) or (ii). Let $x \in X_{m, n} \cap$ Range( $s$ ) with $x \neq r$. We will complete the proof by showing $D(x, r), f(x)$ differ by $<13 / n$.

If (i) holds and $r \in X_{m, n}$, then with $s$ replacing $r$ in Lemma 6 and $r$ replacing $x$, we get that from Lemma 6 (ii) that $D(r, s), f(r)$ differ by less than $5 / n \leq 5$. Combining this with Lemma 6 (i), $D(r, s), f(s)$ differ by at most 9 . Using Corollary 7 we conclude that $D(r, s), f(s)$ differ by less than 9 for all $r$ satisfying (i). Since this property is also part of condition (ii), we have in all cases that $D(r, s), f(s)$ differ by at most 9 .

If $x \notin I$, then from the first paragraph it follows that $D(r, x), D(s, x)$ differ by less than $1 / m \leq 1 / n$. Also, since $x \in X_{m, n}$, we get that $D(s, x), f(x)$ differ by less than $1 / n$. Therefore, $D(r, x), f(x)$ differ by less than $2 / n$ and we are done.

Assume then that $x \in I$. If $r \in \operatorname{cl}\left(X_{m, n}\right)$, then by Lemma 6(iii) and Corollary $7, D(x, r), f(x)$ differ by $<13 / n$ and we are done. This finishes Case (i). We shall assume, therefore, that $r$ satisfies (ii). 
By the shrinking of the interval $I$, we have $\operatorname{rank}(r) \geq m$. If $x$ is in Range $(r)$, then by definition of $X_{m, n}, D(r, x), f(x)$ differ by less than $1 / n$, and we are done. If $x \notin \operatorname{Range}(r)$, then as part of condition (ii), there is some $x^{\prime}$ in $X_{m, n} \cap$ Range $(r)$ between $r, x$. By Lemma 6(iii), $D\left(x, x^{\prime}\right), f(x)$ differ by less than $13 / n$, and $f\left(x^{\prime}\right), f(x)$ differ by less than $8 / n$. By the definition of $X_{m, n}$, $D\left(x^{\prime}, r\right), f\left(x^{\prime}\right)$ differ by less than $1 / n$, and so $D\left(x^{\prime}, r\right), f(x)$ differ by less than $9 / n$. Then, by convexity, we also get that $D(x, r), f(x)$ differ by less than $13 / n$.

Corollary 17. Let $s, m, J$ be as in Lemma 16 and let $n \leq m$. Suppose $K$ is a contiguous interval of $\operatorname{cl}\left(X_{m, n}\right)$ with $\operatorname{cl}(K) \subset J$. Let $r$ be the element of $S \cap \operatorname{cl}(K)$ with smallest rank. Then $r$ is an $(m, i)$-good replacement for $s$ for each $i \leq n$.

Proof. Since $\operatorname{cl}(J) \subset \operatorname{int}(\operatorname{Range}(s))$, it follows that $r \in \operatorname{int}(\operatorname{Range}(s))$ and hence $\operatorname{rank}(r) \geq \operatorname{rank}(s)$. Therefore, if $\operatorname{cl}(K)$ contains $s$, then $r$ must be equal to $s$ and we are done. So assume that $s \notin \operatorname{cl}(K)$. If $r$ is an endpoint of $K$, then $r \in J \cap \operatorname{cl}\left(X_{m, n}\right) \subset J \cap \operatorname{cl}\left(X_{m, i}\right)$ for each $i \leq n$, and by Lemma 16 we are done. Otherwise, let $y$ be the endpoint of $K$ closest to $s$. Then $y \in \operatorname{cl}\left(X_{m, n}\right)$ and is between $r$ and $s$. Also, by choice of $r, y \in \operatorname{int}(\operatorname{Range}(r))$. Choose $y^{\prime} \in X_{m, n}$ so close to $y$ that $y^{\prime}$ is also between $r$ and $s$ and in Range $(r) \cap \operatorname{Range}(s)$. Then $f\left(y^{\prime}\right), f(s)$ differ by less than $4 / n$ by Lemma $6(\mathrm{i})$. Also, by definition of $X_{m, n} D\left(r, y^{\prime}\right), f\left(y^{\prime}\right)$ differ by less than $1 / n$ as do $D\left(y^{\prime}, s\right), f\left(y^{\prime}\right)$. Hence, by convexity, $D(r, s), f\left(y^{\prime}\right)$ differ by less than $1 / n$. Consequently, $D(r, s), f(s)$ differ by less than $5 / n \leq 5$. Also, arbitrarily close to each endpoint of $K$ there exist elements $x^{\prime}$ in $X_{m, n} \cap$ Range $(r) \subseteq X_{m, i} \cap$ Range $(r)$. Therefore, for each $x \notin \operatorname{Range}(r)$ there is an $x^{\prime} \in X_{m, i} \cap \operatorname{Range}(r)$ between $r, x$. Now apply Lemma 16.

Lemma 18. Let $s, m, J$ be as in Lemma 16. Let $p \in J$ be an $m$-good replacement for $s$. Then there are points $q$ arbitrarily close to $p$ on either side which are also m-good replacements for $s$. Furthermore, $q$ can be chosen so that $|D(q, p)-f(p)|$ is as small as we wish.

Proof. By the definition of "good replacement", for each $n \leq m$ there is an $\eta(n)<16 / n$ such that for each $x$ in $X_{m, n} \cap \operatorname{Range}(s)$ with $x \neq p$ we have $D(p, x)-f(x)<\eta(n)$. Let $\epsilon<\min \{16 / n-\eta(n) \mid n=1,2, \ldots, m\}$. Let I be from Lemma 6 applied to $p, m$. Using Corollaries 7, 8, let $L \subset I \cap J$ be a neighborhood of $p$ such that if $q \in L$ with $D(q, p), f(p)$ differing by less than 1 , then $D(q, x)-D(p, x)<\epsilon$, for any $x \in \operatorname{cl}\left(X_{m, 1}\right) \cap \operatorname{Range}(s) \cap \mathrm{c} I$. 
We concentrate on the left of $p$ (the proof on the right of $p$ is similar). Suppose we wish that $D(q, p), f(p)$ differ by less than $1 / w$, where $w \in \mathbb{Z}^{+}$and $w>m$. Let $m^{\prime}$ be large enough that $p \in X_{m^{\prime}, w}$. Let $n$ be the smallest number $n \leq m$ (if there is any) such that $p$ is isolated on the left from $X_{m, n}$. If no such $n$ exists, set $n=m+1$. Let $q \in \operatorname{path}(p) \cap L$ with $q<p, \operatorname{rank}(q)>m^{\prime}$, and $\mathrm{q}$ in the right half of the left-isolating interval (if it exists). Then $D(q, p)$, $f(p)$ differ by less than $1 / w<1 / m$. It remains to find at least one such $q$ which is an $(m, i)$-good replacement for $s$, for each $i \leq m$.

We first consider the case where $1 \leq i<n$. Then by choice of $n, X_{m, n-1}$ has points arbitrarily close to $p$ on the left. If $\mathrm{cl}\left(X_{m, n-1}\right)$ contains a leftneighborhood of $p$, then we may also choose $q \in \operatorname{cl}\left(X_{m, n-1}\right)$. But if $\operatorname{cl}\left(X_{m, n-1}\right)$ contains no such neighborhood, choose $q$ to be the element of smallest rank inside some contiguous interval $K$ of $\operatorname{cl}\left(X_{m, n-1}\right)$, with $\operatorname{cl}(K) \subseteq J$. In either case, (by Lemma 16(i) or Corollary 17 resp.) $q$ is an $(m, i)$-good replacement for $s$. This concludes the case $1 \leq i<n$, and hence also concludes the case where $n>m$. Hence we may assume that $p$ is isolated on the left from $X_{m, n}$.

We now consider the case $n \leq i \leq m$ and let $x \in X_{m, i} \cap$ Range $(s)$ with $x \neq q$. We will complete the proof by showing $D(q, x), f(x)$ differ by less than $\max (\eta(i)+\epsilon, 15 / i)$ which is less than $16 / i$.

If $x=p$, then since $D(q, p), f(p)$ differ by less than $1 / w<1 / m$ we are done. So assume $x \neq p$.

If $x \notin I$, then $D(q, x), D(p, x)$ differ by less than $\epsilon$ and using that $p$ is an $(m, i)$-good replacement for $s, D(p, x), f(x)$ differ by less than $\eta(i)$. Hence $D(q, x), f(x)$ differ by less than $\eta(i)+\epsilon$ and we are done. So assume $x \in I$.

Since $D(q, p), f(p)$ differ by less than $1 / w<1 / i$ and by Lemma $6(\mathrm{i}), f(p)$ and $f(x)$ differ by less than $4 / i$, then $D(q, p), f(x)$ differ by less than $5 / i$. Also, $D(p, x), f(x)$ differ by less than $5 / i$ by Lemma $6(\mathrm{ii})$.

Case 1: $x>p$. Then by convexity, $D(q, x), f(x)$ differ by less than $5 / i$.

Case 2: $x<p$. Since $q$ is in the right half of the interval isolating $p$ on the left from $X_{m, n}$, and since $X_{m, i} \subseteq X_{m, n}, q$ must be closer to $p$ than it is to $x$. It follows by Lemma 5 , that $D(x, q), f(x)$ differ by less than $15 / i$.

Lemma 19. Let $s, m, J$ be as in Lemma 16 and let $n \leq m$. If $s^{*} \in J$ is an $m$-good replacement for $s$ and $s^{*}$ is an $(m, i)$-very good replacement for $s$ for each $i$ such that $n<i \leq m$, then there is an $s^{* *}$ arbitrarily close to $s^{*}$, with $F\left(s^{* *}\right)$ arbitrarily close to $F\left(s^{*}\right)$ such that $s^{* *}$ is an $m$-good replacement for $s$ and $s^{* *}$ is an $(m, i)$-very good replacement for $s$ for each $i$ such that $n \leq i \leq m$.

Proof. Since $s^{*} \in J, s^{*}$ is in $\operatorname{int}(\operatorname{Range}(s))$. If $s^{*} \notin \operatorname{cl}\left(X_{m, n}\right)$, then by Lemma $15, s^{*}$ is an $(m, n)$-very good replacement for $s$ and letting $s^{* *}=s^{*}$ 
we are done. Also, if $X_{m, n}$ is dense in a neighborhood of $s^{*}$, then again $s^{*}$ is an $(m, n)$-very good replacement for $s$ (by Lemma 16(i)), and we are done. If $s^{*}$ happens to be isolated on either the left or right from $\operatorname{cl}\left(X_{m, n}\right)$, then by Lemma 18, choose $s^{* *} \in \operatorname{int}(\operatorname{Range}(s)) \backslash \operatorname{cl}\left(X_{m, n}\right)$ so that $s^{* *}$ is also an $m$-good replacement for $s$, and such that the difference between $s^{*}$ and $s^{* *}$ and also between $F\left(s^{*}\right)$ and $F\left(s^{* *}\right)$ is as small as we wish. Since $\operatorname{cl}\left(X_{m, i}\right) \subset \operatorname{cl}\left(X_{m, n}\right)$ for each $i \geq n$, by Lemma $15 s^{* *}$ is an $(m, i)$-very good replacement for $s$, and we are done.

Therefore, we may assume that there are contiguous intervals of $\operatorname{cl}\left(X_{m, n}\right)$ arbitrarily close to $s^{*}$. Let I be from Lemma 6 applied to $s^{*}, m$. Reduce I if necessary so that no element of $S \cap I \backslash\left\{s^{*}\right\}$ has rank $\leq m$. Choose an interval $K \subset J$ which is contiguous to $\operatorname{cl}\left(X_{m, n}\right)$ and which is close enough to $s^{*}$ that $\operatorname{cl}(K) \subset J \cap I$. For each $i$ such that $n<i \leq m, s$ is an $(m, i)$-good replacement for $s$. Therefore, we may choose $K$ so close to $s^{*}$ that whenever $r \in \operatorname{cl}(K)$ with $D\left(r, s^{*}\right), f\left(s^{*}\right)$ differing by less than 10 we have that $r$ is an $(m, i)$-good replacement for $s$. Let $p$ be in $S \cap \operatorname{cl}(K)$ of minimal rank. Then by Corollary 17, $p$ is $(m, i)$-good for $s$ for all $i \leq n$.

If $p \in X_{m, n}$, then by Lemma $6(\mathrm{i}), f(p), f\left(s^{*}\right)$ differ $b y<4 / n$ while by Lemma 6 (ii), $D\left(p, s^{*}\right), f(p)$ differ by $<5 / n$, and so $D\left(p, s^{*}\right) f\left(s^{*}\right)$ differ by $<9 / n$ which is less than 10 .

If $p \notin X_{m, n}$, let $x$ be the endpoint of $K$ closest to $s^{*}$. Then $x \in \operatorname{cl}\left(X_{m, n}\right)$. If $x \in X_{m, n}$, leave it alone. Otherwise, move it a little closer to $s^{*}$ so that it is in $X_{m, n}$, but still in Range $(p)$. In either case, $x \in X_{m, n} \cap \operatorname{Range}(p)$. Then $D(p, x), f(x)$ differ by less than $1 / n$. By Lemma $6(\mathrm{i}), f(x), f\left(s^{*}\right)$ differ by less than $4 / n$. By Lemma 6 (ii), $D\left(x, s^{*}\right), f(x)$ differ by less than $5 / n$. So by convexity, $D\left(p, s^{*}\right), f(x)$ differ by less than $5 / n$. Therefore, we have again that $D\left(p, s^{*}\right), f\left(s^{*}\right)$ differ by less than $9 / n<10$.

So in either case, $p$ is an $(m, i)$-good replacement for $s$ for each $i$ such that $n<i \leq m$. It follows that $p$ is an $m$-good replacement for $s$.

By Lemma 18, there must be some $s^{* *}$ in $\operatorname{int}(K)$ which is also an $m$-good replacement for $s$. By the definition of $K, s^{* *} \notin \operatorname{cl}\left(X_{m, n}\right)$. If $n \leq i \leq m$, then also $s^{* *} \notin \operatorname{cl}\left(X_{m, i}\right)$. Also, by Lemma 16, $J \subseteq \operatorname{int}(\operatorname{Range}(s))$; so $s^{* *} \in K \subseteq J \subseteq$ $\operatorname{int}($ Range $(s))$. Hence by Lemma $15, s^{* *}$ is an $(m, i)$-very good replacement for $s$.

Since $s^{* *}$ can be chosen arbitrarily close to $p$ and $p$ arbitrarily close to $s^{*}$, we have $s^{* *}$ arbitrarily close to $s^{*}$. As part of Lemma $18, F\left(s^{* *}\right)$ can be chosen arbitrarily close to $F(p)$, and since $D\left(p, s^{*}\right)$ differs from $f\left(s^{*}\right)$ by less than $10, F(p)$ can be chosen arbitrarily close to $F\left(s^{*}\right)$. Therefore, $F\left(s^{* *}\right)$ can be chosen arbitrarily close to $F\left(s^{*}\right)$ and we are done. 
Lemma 20. Let $s, m$, be as in Lemma 16. Then there are points $t \in T$ which are arbitrarily close to $s$ on either side, with $|D(s, t)-f(s)|$ arbitrarily small, such that $t$ is an m-good replacement for $s$.

Proof. Let $J$ also be as in Lemma 16. Suppose we wish that $D(s, t), f(s)$ differ by less than $1 / w$. Trivially, $s$ is an $m$-good replacement for itself. Therefore, by Lemma 18, let $s_{0}<s$ be such that $s_{0} \in J$ and $s_{0}$ is an $m$-good replacement for $s$ and such that $D\left(s, s_{0}\right), f(s)$ differ by less than $1 / w$. We are not done, however, since $s_{0}$ might not be in $T$. Now it is valid to apply Lemma 19 with $n=m$ to find a nearby $s_{1} \in J$ with $s_{1}<s$, and choose $s_{1}$ so close to $s_{0}$, with $F\left(s_{1}\right)$ so close to $F\left(s_{0}\right)$ that $D\left(s_{1}, s\right), f(s)$ still differ by less than $1 / w$ and such that $s_{1}$ is an $m$-good replacement for $s$ and also $(m, m)$ very good for $s$. Apply Lemma 19 again to find a nearby $s_{2} \in J$ with $s_{2}<s$ and $D\left(s_{2}, s\right), f(s)$ differing by less than $1 / w$ and such that $s_{2}$ is an $m$-good replacement for $s$ and for $i=m$ or $i=m-1, s_{2}$ is $(m, i)$-very good for $s$. Continue until $s_{m}<s$ is found which is $m$-good for $s$ and for each $1 \leq i \leq m$, $s_{m}$ is an $(m, i)$-very good replacement for $s$ with $D\left(s_{m}, s\right), f(s)$ still differing by less than $1 / w$. Then using Corollary 9 and Definition 14 find $t \in T$ so close to $s$, with $F(t)$ so close to $F(s)$ that $t<s$ and $t$ is an $m$-good replacement for $s$, and $D(t, s), f(s)$ differ by less than $1 / w$. The argument on the right of $s$ is identical.

Lemma 21. The theorem holds for some dense subset $T^{\prime} \subset T$. That is, there is a trajectory $\tau: T^{\prime} \rightarrow \mathbb{Z}^{+}$such that for each $x$, the $\tau$-first return derivative of $F$ at $x$ is $f(x)$.

Proof. List the elements of $S$ in order of rank, $S=\left(s_{1}, s_{2}, \ldots\right)$. We construct the ordered set $T^{\prime}$ and its ordering $\tau$ in stages. Suppose that at stage $n-1$, each $s_{i}(i<n)$ is associated with $n-i$ elements of $T$ and these are ordered $\left(t_{1}, t_{2}, \ldots, t_{n(n-1) / 2}\right)$ partially creating a new path system. Suppose also that for each $x$, if $t_{j} \in \operatorname{newpath}(x)$ and $s_{i} \neq x$ is associated with $t_{j}$, then $s_{i} \in$ $\operatorname{path}(x)$.

Stage $n$ : With each $s_{i}(i<n)$ choose a new $t \in T$ to associate with it such that $t$ is between $s_{i}$ and $s_{n}$ and is closer to $s_{i}$ than any previously chosen $t^{\prime} \in T$ with $t^{\prime} \neq s_{i}$. Number these new elements of $T, t_{n(n-1) / 2+1}, \ldots, t_{n(n-1) / 2+(n-1)}$ in the order of the $s_{i}^{\prime} s$ which they are associated with. Now associate with $s_{n}$ a new element $t_{n(n+1) / 2} \in T$ closer to $s_{n}$ than any previously chosen $t\left(t \neq s_{n}\right)$.

Claim: If $t_{j} \in \operatorname{newpath}(x)$ is associated with $s_{i} \neq x$, then $s_{i} \in \operatorname{path}(x)$. Proof of Claim: If $s_{i} \notin \operatorname{path}(x)$, then there is some $k<i$ such that $s_{k}$ is between $s_{i}$ and $x$. Then at stage $i$ there is a $t^{\prime}$ associated with $s_{k}$ between $s_{k}$ and $s_{i}$. Then $t^{\prime}$ is between $s_{i}$ and $x$. Since $t_{j}$ is not associated before stage $i$ 
and therefore is associated after $t^{\prime}$, we can't have $t^{\prime}$ between $t_{j}$ and $s_{i}$. Then since $t^{\prime}$ is between $s_{i}$ and $x$, it must be that $t^{\prime}$ is between $t_{j}$ and $x$ contradicting that $t_{j} \in \operatorname{newpath}(x)$. This finishes the proof of the claim.

By Lemma 20, for each $s_{m}$, with bounded range, each $t_{j} \in T$ associated with it can be chosen to be an $m$-good replacement for $s_{m}$ with $D\left(t_{j}, s_{m}\right)$, $f\left(s_{m}\right)$ differing by less than $1 / j$. Fix $n^{\prime}$ and let $x \in \mathbb{R}$. Let $n=16 n^{\prime}$ and choose $m$ so that $m>n$ and $x \in X_{m, n}$. Let $m^{\prime}>m$ be such that by stage $m^{\prime}$ there exists $u, v \in T$ with $u<x<v$ where $u$ is associated with some $s_{i}<u$ and $v$ is associated with some $s_{k}>v$ and where both $s_{i}$ and $s_{k}$ are in $\operatorname{path}(x)$ with bounded range, and both $i, k$ are greater than $m$. Let $t_{j} \in \operatorname{newpath}(x)$ with $j>m^{\prime}\left(m^{\prime}+1\right) / 2$. We will complete the proof by showing that $D\left(t_{j}, x\right)$, $f(x)$ differ by less than $1 / n^{\prime}$. Now $t_{j}$ is associated with some $s_{z}$ and is chosen after both $u$ and $v$. If $s_{z}=x$, then $D\left(t_{j}, x\right), f(x)$ differ by less than $1 / j<1 / n^{\prime}$ and we are done. So assume $s_{z} \neq x$. Since $t_{j}$ is between $u, v$, it follows that $s_{z}$ is between $u$ and $v$ (inclusive) and hence (strictly) between $s_{i}$ and $s_{k}$. Since both $s_{i}$ and $s_{k}$ are in $\operatorname{path}(x)$, it must be that $z>m$. Also by the claim, $s_{z} \in \operatorname{path}(x)$. Then, since $t_{j}$ is a $z$-good replacement for $s_{z}$, and $x \in X_{m, n} \subset X_{z, n}$, we have that $D\left(t_{j}, x\right)$ and $f(x)$ differ by less than $16 / n=1 / n^{\prime}$.

Lemma 21 solves the problem for the case where the new support set is

allowed to be a certain subset of the target set $T$. By following Lemma 21 with an application of Lemma 13 the proof of the Theorem is completed.

\section{References}

[1] U. B. Darji, M. J. Evans, and R. J. O'Malley, Some interesting small subclasses of the Darboux Baire 1 functions, Real Analysis Exchange, 19 (1993-94), 328-331.

[2] U. B. Darji, M. J. Evans, and R. J. O'Malley, First return path systems: differentiability, continuity, and orderings, Acta Math. Hungar. 66 (1995), 83-103.

[3] U. B. Darji, M. J. Evans, and R. J. O'Malley, Universally first return continuous functions, Proc. Amer. Math. Soc. 123, (1995), 1727-1736.

[4] M. J. Evans, First return differentiation, Report on the Joint U.S. - Polish Real Analysis Workshop, Real Analysis Exchange 20 (1994-95), 441-442. 